\title{
A framework for time-driven activity-based costing implementation at small and medium enterprises
}

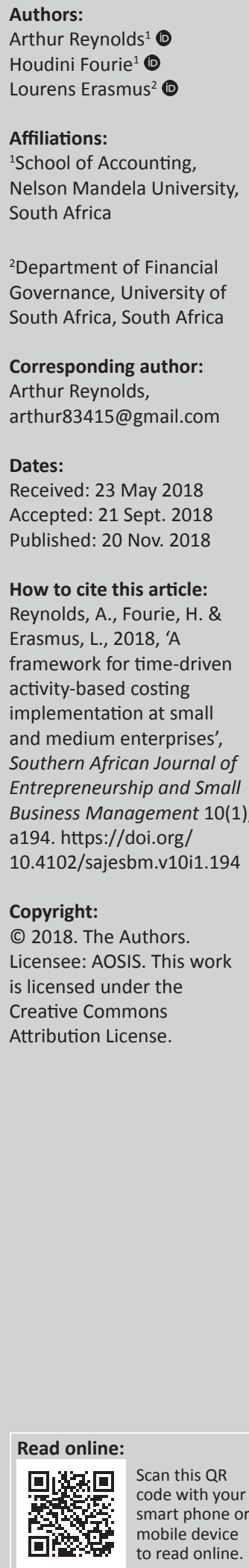

Background: South African small and medium enterprises (SMEs) are struggling to survive because of limited resources and access to low-cost funding. It may be beneficial for SMEs to focus on cost management within their organisation to mitigate the impact of inflationary pressure and high-cost funding. Published literature provides evidence that time-driven activity-based costing (TDABC), leaning on the theory of constraints (TOC), can be implemented successfully at SMEs within different industries. Furthermore, it was demonstrated how TDABC can be effective when evaluating product cost for a wide, but fixed, product range. There is, however, no generic framework available in extant literature on how to implement TDABC effectively at SMEs.

Aim: The purpose of the study was to devise a framework for SMEs with resource constraints to be able to implement TDABC without exhausting limited resources.

Setting: A case study at a manufacturing SME specialising in custom products was employed.

Methods: The generic steps for TDABC as described in published literature were adapted for the manufacturing SME where the case study was conducted.

Results: The study found that it was practical to implement TDABC at a manufacturing SME by extrapolating the cost data from a single product which harnesses all the key activities in the factory. The TDABC costing data were sufficient for analysis of unused capacity and overexpenditure, hence providing information to consider in profitability enhancement and to support the TOC.

Conclusion: The proposed framework for TDABC implementation at SMEs revises the generic framework suggested in literature by focusing on key products only and consolidating activities.

\section{Introduction}

The prospects of survival for small and medium enterprises (SMEs) in South Africa appear to be unfavourable. Olawale and Garwe (2010:729) suggest that up to 75\% of SMEs in South Africa do not survive in the long term. Substandard infrastructure, high overheads and difficulty in obtaining financing are some of the factors that hamper the sustainability of SMEs (Ingle 2014:40; National Credit Regulator [NCR] 2011). Although there will be external factors outside the control of the typical entrepreneur, it may be beneficial for SMEs to focus on managing costs within their organisation, thereby mitigating the impact of high-cost funding and inflationary pressure. If there is an opportunity to highlight over-expenditure, it may be worthwhile to consider the costing systems that may assist SMEs in reducing costs and managing their cash flow; not only in South Africa but also worldwide.

Drury (2015:259) has identified three types of costing systems commonly used in all industries, namely: direct costing, absorption costing and activity-based costing ( $A B C)$. If effectively implemented, $\mathrm{ABC}$ could provide more accurate product cost information than direct or absorption costing (Akyol, Tuncel \& Bayhan 2005:139). The concern with ABC implementation is that intensive implementation may be counterproductive and may require too many resources (Reynolds \& Van der Poll 2015:129). In published literature, the successful implementation of ABC at SMEs was shown to be possible, although it required modification of the general principles of the costing system (Shea et al. 2018). 
A further costing system, not mentioned by Drury (2015), is the closely related time-driven activity-based costing (TDABC) introduced by Kaplan and Anderson (2003). Kaplan and Anderson (2003:5) maintain that TDABC offers benefits over traditional $\mathrm{ABC}$ by being less complex. The application of TDABC enables organisations to consume fewer resources, whilst also calculating unused capacity (Kaplan \& Anderson 2003:1). Kaplan and Anderson (2003:15) believe that similar benefits could be accrued from the less complex TDABC, which suggests that it may be a suitable costing system for SMEs with resource constraints. Many scholars found that organisations could benefit from TDABC if the limitations of the organisation are considered (Balakrishnan, Goico \& Arjmand 2015; Fladkjaer \& Jensen 2011; Musov 2017; Stouthuysen et al. 2014). Furthermore, it has been demonstrated that a lack of resources associated with SMEs is not a hindrance to TDABC implementation (Fladkjaer \& Jenson 2011; Ganorkar, Lakhe \& Agrawal 2018; Somapa, Cools \& Dullaert 2012). However, the challenges that SMEs and organisations with limited resources face when implementing TDABC, suggest that the generic TDABC process steps (detailed later) prescribed by Kaplan and Anderson (2003) may be insufficient for many SMEs to achieve the desired benefits.

The main problem identified by the current study was the unsuitability of the generic TDABC process steps for SMEs with limited resources. Furthermore, the survival rate for SMEs is low and there is a need for SMEs to quantify the cost implications with the transparency that TDABC is supposed to offer.

The primary objective of the current study was to develop a framework for the implementation of TDABC at manufacturing SMEs that may assist resource-constrained organisations in optimising costs. The current study specifically focused on implementing TDABC at a manufacturing SME, specialising in custom products using minimal resources.

To achieve the research objective, a literature review and a TDABC implementation case study were conducted. A conceptual and literature framework for the research was formulated, which is discussed below.

\section{Conceptual and literature framework}

Many SMEs are facing significant challenges to manage their resources and maintain profitability. The theory of constraints (TOC) dictates that resources can be managed by eliminating the weakest area within any given system (Mabin 2015:131). The TOC uses a five-step process to continually manage constrained resources until the constraint is resolved (Goldratt 1990:6-7). The use of TOC at a manufacturing organisation can improve production throughput and ensure on-time delivery (Pegels \& Watrous 2005:302). It is plausible that a transparent costing system, such as TDABC, may be able to highlight the areas of constraint. Therefore, the current study is based on the premise that TDABC could support TOC.

As mentioned earlier, the implementation of TDABC may require fewer resources than the closely related $\mathrm{ABC}$ and may, therefore, be suitable for SMEs. There are six basic steps that could be identified in literature (Figure 1). TDABCis based on two key variables, namely, unit cost of capacity supplied and unit time estimate (Kaplan \& Anderson 2003:6).

The first step to TDABC implementation is the identification of relevant activities and resource objects (Kaplan \& Anderson 2003:6; Stouthuysen et al. 2014:290). The resource groups may include items such as wages and energy costs (Stouthuysen et al. 2014:293).

The second step entails the calculation of total activity costs (Kaplan \& Anderson 2003:6; Stouthuysen et al. 2014:290). The calculation of total activity costs may be achieved by estimating or calculating how resources are consumed per activity (Stouthuysen et al. 2014:291-292). In the study of Stouthuysen et al. (2014) for TDABC implementation at a public swimming pool, the resources (totalling R906 938) were allocated on a percentage basis (Table 1 ).

From the information in Table 1, it can be derived that the swimming activity consumed $75 \%$ of energy cost, as it entails using the swimming pool. Conversely, the supervision of swimmers requires an employee (lifesaver), but does not consume other resources.

The third step requires an estimation of the practical capacity (Kaplan \& Anderson 2003:6; Kee 2012:39; Stouthuysen et al. 2014:290). Practical capacity (as a percentage) can be calculated by considering lunch breaks, sick leave and public holidays (Öker \& Adıgüzel 2010:80). In practice, a practical capacity of $80 \%$ is often assumed as a starting point with lack of sufficient data (Askarany \& Franklin-Smith 2014:67; Siquenza-Guzman, Van den Abbeele \& Cattrysse 2014:168). This may be appropriate for SMEs with resource constraints.

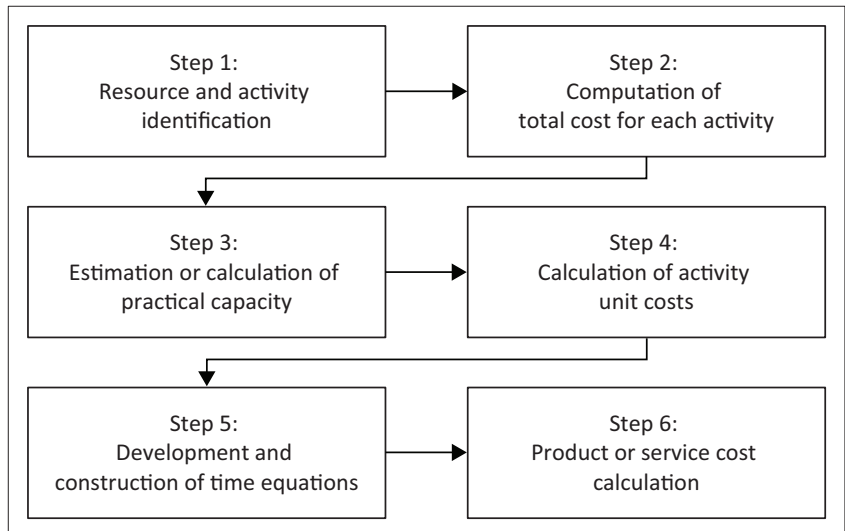

Source: Adapted from Kaplan, R.S. \& Anderson, S.R., 2003, Time-driven activity-based costing, viewed 22 May 2018, from https://papers.ssrn.com/sol3/papers.cfm?abstract id $=485443$ and Stouthuysen, K., Schierhout, K., Roodhooft, F. \& Reusen, E., 2014, 'Timedriven activity-based costing for public services', Public Money \& Management 34(4), 289-296. https://doi.org/10.1080/09540962.2014.920202

FIGURE 1: Generic steps for time-driven activity-based costing implementation. 
TABLE 1: Allocation of resource cost to activities.

\begin{tabular}{|c|c|c|c|c|c|c|}
\hline Resources & Reception & Changing clothes 1 & Swimming & Supervision & Changing clothes 2 & Admin function \\
\hline Wages & $12.0 \%$ & $2.7 \%$ & $9.8 \%$ & $58.3 \%$ & $3.2 \%$ & $14.0 \%$ \\
\hline Energy & $1.0 \%$ & $10.0 \%$ & $75.0 \%$ & $0.0 \%$ & $13.0 \%$ & $1.0 \%$ \\
\hline Maintenance & $0.0 \%$ & $37.5 \%$ & $25.0 \%$ & $0.0 \%$ & $37.5 \%$ & $0.0 \%$ \\
\hline Overhead & $10.0 \%$ & $30.0 \%$ & $30.0 \%$ & $0.0 \%$ & $30.0 \%$ & $0.0 \%$ \\
\hline Depreciation & $10.0 \%$ & $15.0 \%$ & $55.0 \%$ & $0.0 \%$ & $15.0 \%$ & $5.0 \%$ \\
\hline Total cost & R 78107 & R 77335 & R 231449 & R 347613 & R 85284 & R 87151 \\
\hline
\end{tabular}

Source: Adapted from Stouthuysen, K., Schierhout, K., Roodhooft, F. \& Reusen, E., 2014, 'Time-driven activity-based costing for public services', Public Money \& Management 34(4), 289-296. https://doi.org/10.1080/09540962.2014.920202

To calculate the unit cost of capacity supplied (step 4), it is necessary to establish the total (period) cost for a specific activity and the practical capacity (in minutes) (Kaplan \& Anderson 2003:6). The practical capacity (in minutes) is calculated based on the percentage calculated in the previous step (Kaplan \& Anderson 2003:6).

The next step in the TDABC calculation entails the calculation of activity rates for all activities, by multiplying the activity rate, calculated in the first step, with the activity time observed for the activity. To illustrate: in a customer service example from Kaplan and Anderson (2003), the activity credit checks take 250 min each to complete, whilst the minute rate is calculated as R0.80 per minute. Therefore, each credit check is expected to cost $250 \mathrm{~min} \times \mathrm{R} 0.80=$ R200.00 using TDABC (Kaplan \& Anderson 2003:8). In more complicated scenarios, it is necessary to create time equations to allow for special activities (Kaplan \& Anderson 2003:9) constituting step five.

The sixth and final step is to calculate the product (or service) cost by multiplying the activity unit time with the activity cost rates and summating all the activity costs that are relevant (Kaplan \& Anderson 2003:8). The implementation of TDABC at SMEs with resource constraints in South Africa, which use these generic TDABC as described in published literature with the six-step process (depicted in Figure 1), may have to be critically assessed and reviewed in practice.

Published literature suggests that intensive implementation of TDABC at some organisations may not be practical and that compromises may likely have to be made. This may entail the measurement of only one resource group, such as employee cost in the example of TDABC implementation in an operating room (Balakrishnan et al. 2015:6). In addition, it is stated that the measurement of joint support functions, such as maintenance and other fixed costs, is not compulsory and may be excluded for the TDABC calculation, although this could affect the performance of the costing system (Stouthuysen et al. 2014:295). Furthermore, the use of time equations across the product range because of potentially inconsistent characteristics could prove to be difficult to implement (Souza et al. 2010:68). In addition, calculating practical capacity at SMEs may be challenging because of difficulties with availability of relevant data (Fladkjaer \& Jenson 2011). The effectiveness of TDABC can be reduced further if a capable enterprise resource system is not present (Stout \& Propri 2011). However, using a spreadsheet solution for TDABC analysis, as proposed by Fladkjaer and Jenson (2011), could potentially be effective and is likely to be the only option for many SMEs.

\section{Research methods and design}

The current study was conducted using a case study approach and represents the implementation phase prior to a 3-month evaluation programme. A comprehensive literature review was conducted to obtain background to the basic TDABC implementation steps at a manufacturing SME. The manufacturing SME used for the case study was obtained by means of convenience sampling.

The manufacturing SME that participated in the study specialises in polyvinyl chloride (PVC) and canvas products. The company is based in Port Elizabeth in the Eastern Cape Province of South Africa and mainly manufactures custommade products for its customers. A large variety of custommade products distinct from one another are available, namely, tarpaulins, truck curtains, canopy covers, cargo nets, boat covers, sun canopies, box covers and outdoor blinds, amongst others. The SME employs around 20 people and the factory is housed in a $1814 \mathrm{~m}^{2}$ factory building on a rental agreement. The organisation requested to be anonymous and requested the researchers to refrain from divulging sensitive financial information in publications. Therefore, the organisation is not named and minimum financial information is presented, except in the context of illustrative TDABC computations.

\section{Data collection methods}

The data were collected mainly by analysis of documentary evidence, supported by direct observations, time studies and informal interviews. Documentary evidence entailed the data collection of stock listings, financial statements, insurance agreements, factory layouts and invoice data from the accounting system (Pastel). Direct observations were used to measure floor space, review the number of employees per activity and observe the production and non-production work steps. Time studies were used to determine the duration of the production work steps. Informal interviews were held with the owner, as well as staff members, to establish the production workflow, basic assumptions and work step times. The results from the TDABC implementation were confirmed with the owner at a later stage by means of a semistructured interview relating to the accuracy of headcount, floor space and time study parameters. 


\section{Parameters for time-driven activity- based costing system}

The first step was to consider the resource groups to be used for the TDABC calculation. After review of the financial statements, seven resource groups were identified, namely, salaries and wages, energy, telephone expenses, insurance, rental, vehicle expenses and other overheads. The allocation of costs for the TDABC calculation was conducted accordingly (Table 2). Salaries and wages, telephone expenses and vehicle expenses could initially be assigned to the actual split per activity because information was available, although not necessarily easy, to extract, because of the lack of cost centres. For further evaluations, a reasonable approximation was considered sufficient for TDABC calculations. It was deemed appropriate to use floor space for rental and insurance. The remainder of the resources was assigned depending on the number of employees (man-hours) performing each activity.

The next step was to consider the primary activities associated with the company. After consultation with the owner of the company, six primary activities were identified, namely, cutting, welding, stitching, sales, administration and fitting. The cutting activity entails cutting of material (e.g. PVC or canvas) to the correct specification and also incorporates quality inspection of products. The welding activity receives the material from the cutting department and welds additional material, such as windows and other protection, onto the received material. The stitching activity adds zips and other material by means of stitching, using sewing machines. Sales entail the activities of invoicing and customer support. The administration function includes the management and bookkeeping activities of the company.

TABLE 2: Resource cost allocation method.

\begin{tabular}{ll}
\hline Resource & Cost allocation \\
\hline Wages and salaries & Specific split \\
Energy & Number of people (man-hours) \\
Telephone expense & Specific split \\
Insurance & Floor space \\
Rental & Floor space \\
Vehicle expense & Specific split \\
Other overheads & Number of people (man-hours) \\
\hline
\end{tabular}

The six activities incorporated numerous work steps which will be described in detail later.

To calculate the floor space for each activity, it was necessary to obtain a layout of the factory (Appendix 1) and calculate the floor space by physical measurement of each area. A floor space matrix was utilised to allocate activities to the different areas. The use of a matrix was required as many areas were shared amongst the various activities. The primary areas were allocated first and the remainder of the areas were then allocated, specifically or proportionally, after consultation with the SME owner (Table 3).

The total number of employees $(N=22)$ at the company were allocated to the six activities: cutting $(n=7)$, welding $(n=2)$, stitching $(n=5)$, sales $(n=4)$, administration $(n=2)$ and fitting $(n=2)$. The number of employees in the cutting department was later reduced to five people $(n=5$; total headcount $N=20$ ) because of reduced orders, which is reflected in the evaluation of the costing data. It was evident from the computation that the stitching department was housed in a disproportionately large area with $41.8 \%$ of the total area and only $25 \%$ of the work force $(n=5)$ utilising the area. To assign practical capacity for each activity, it was assumed that an arbitrary $80 \%$ of total capacity is available and that each employee works a total of $8 \mathrm{~h}$ per day. It was not considered practical to physically measure the actual capacity of each employee. Therefore, each employee was assumed to be able to work productively (at $80 \%$ efficiency) for a total of $6.4 \mathrm{~h}$ $(8 \mathrm{~h} \times 80 \%)$ per day. Work days were from Monday to Friday and overtime was not considered. The practical capacity (in minutes) was calculated by multiplying the number of available hours with the number of working days (Table 4). In addition to calculating the cost rates for each activity, the cost rate per minute was calculated. It was calculated by dividing the total resource costs assigned to the activity (in Rand) by the practical capacity (in minutes) to obtain a cost per minute rate. In addition, the cost per minute rate for each of the resource groups could be calculated, for example, in the sales activity: the total cost rate is $\mathrm{R} 2.79$ per minute constituting: salaries and wages (R1.62 per minute), energy (R0.05 per minute), telephone (R0.10 per minute), insurance (R0.05 per minute), rental (R0.21 per minute), vehicle expense

TABLE 3: Floor space allocation matrix (presented in $\mathrm{m}^{2}$ ).

\begin{tabular}{|c|c|c|c|c|c|c|c|}
\hline Department & Cutting & Welding & Stitching & Sales & Admin & Fitting & Totals \\
\hline Primary area & 348.96 & 130.87 & 599.65 & 58.46 & 32.35 & 35.99 & 1206.29 \\
\hline Off-loading bay & - & - & - & 109.30 & - & - & 109.30 \\
\hline Storeroom 1 & - & - & - & 30.34 & - & - & 30.34 \\
\hline Storeroom 2 & - & - & - & 33.90 & - & - & 33.90 \\
\hline Storeroom 3 & - & - & - & 31.08 & - & - & 31.08 \\
\hline Strong room & - & - & - & - & 23.01 & - & 23.01 \\
\hline Meeting room & 9.84 & 3.69 & 16.92 & 7.42 & 1.56 & 1.02 & 40.45 \\
\hline Men's toilets & 13.23 & 4.96 & 22.73 & 9.97 & 2.10 & 1.36 & 54.36 \\
\hline Ladies toilets & 4.39 & 1.65 & 7.55 & 3.31 & 0.70 & 0.45 & 18.05 \\
\hline Tenant space & 43.21 & 16.21 & 74.25 & 32.57 & 6.86 & 4.46 & 177.55 \\
\hline Vacant space & 16.50 & 6.19 & 28.35 & 12.44 & 2.62 & 1.70 & 67.80 \\
\hline Total & 441.36 & 165.53 & 758.44 & 332.73 & 70.02 & 45.52 & 1813.60 \\
\hline
\end{tabular}

$m^{2}$ square metre. 
TABLE 4: Practical capacity and cost rates for each activity.

\begin{tabular}{|c|c|c|c|c|c|c|}
\hline Activities & Cutting & Welding & Stitching & Sales & Admin & Fitting \\
\hline No. of employees & 5 & 2 & 5 & 4 & 2 & 2 \\
\hline Working hours & 8 & 8 & 8 & 8 & 8 & 8 \\
\hline Capacity \% & 80 & 80 & 80 & 80 & 80 & 80 \\
\hline Available daily hours & 6.40 & 6.40 & 6.40 & 6.40 & 6.40 & 6.40 \\
\hline No. of days & 21 & 21 & 21 & 21 & 21 & 21 \\
\hline Total capacity (in minutes) & 40320 & 16128 & 40320 & 32256 & 16128 & 16128 \\
\hline Cost per minute (in Rand) $\dagger$ & $1.51 \dagger$ & $1.86 \dagger$ & $1.68 \dagger$ & $2.79 \dagger$ & $4.61 \dagger$ & $1.61 \dagger$ \\
\hline Salaries and wages & 0.93 & 1.21 & 0.87 & 1.62 & 2.99 & 0.87 \\
\hline Energy & 0.03 & 0.04 & 0.03 & 0.05 & 0.09 & 0.03 \\
\hline Telephone expense & 0.00 & 0.00 & 0.00 & 0.10 & 0.03 & 0.01 \\
\hline Insurance & 0.06 & 0.05 & 0.10 & 0.05 & 0.02 & 0.01 \\
\hline Rental & 0.22 & 0.20 & 0.37 & 0.21 & 0.09 & 0.06 \\
\hline Vehicle expense & 0.00 & 0.00 & 0.00 & 0.23 & 0.52 & 0.33 \\
\hline
\end{tabular}

$\dagger$, The cost per minute rate is calculated from total resource costs (in Rand) and the total capacity (in minutes)

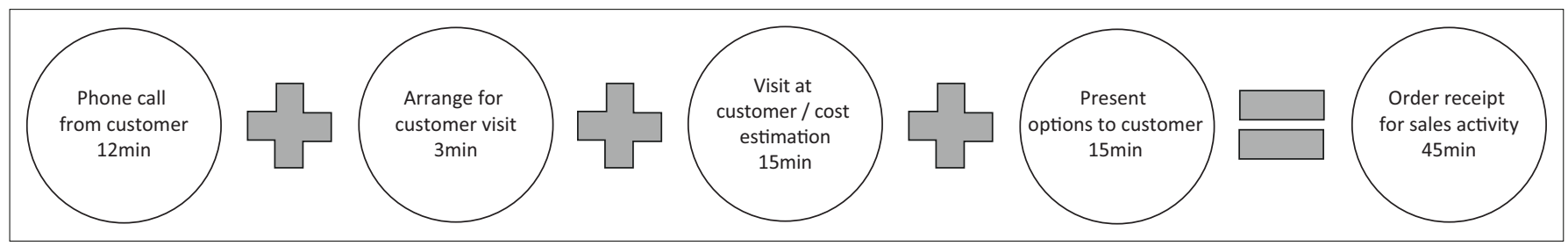

FIGURE 2: Allocation of work steps to sub-activity.

(R0.23 per minute) and other overheads (R0.53 per minute). In this evaluation, it was already evident that administration carries a high labour rate, the stitching department incurs high rental costs because of large floor space allocation and the sales and administration department have vehicle expenses to consider that were not prevalent in other activities.

A complete implementation of TDABC, that would consider each product, was considered impractical for a company of this size and it was necessary to find an alternative solution. After consultation with the owner, it was assessed that the only custom-made product that sells on a regular basis and includes all the activities were PVC blinds. It was, therefore, decided to focus the TDABC implementation on this product only. It should be noted that the PVC blinds can differ significantly in size and specification. The next step was to evaluate the PVC blinds to establish how resources will be consumed for the TDABC system.

\section{Calculation of resources consumed for polyvinyl chloride blinds}

The TDABC calculation used for this study was conducted in Microsoft Excel for Mac. As explained earlier, the TDABC was applied for one product only and results were then extrapolated. The PVC blinds were selected because of their popularity and because their manufacturing encompassed all six activities. The PVC blinds represented around 5\% of total sales during the evaluation phase. The first step in the evaluation of the PVC blinds manufacturing process was to establish the working steps required to produce a PVC blind across the value chain, from receipt of order to delivery to the customer. A total number of 44 work steps were identified (Appendix 2). Each work step was assigned to an activity, allocated a relevant cost driver and duration. The duration for each work step was assigned in two ways, namely, consultation with the relevant person and a time study for the production-related work steps. The cost drivers and time durations were confirmed with the owner as accurate after data collection.

An extra column was added to each work step to further categorise each work step into one of 13 sub-activities (Appendix 3). To illustrate how a sub-activity was created, the order receipt for a sales activity can be used (Figure 2). There are four work steps related to this activity, namely, call from the customer (12 min), arrangements to go to customers' place (3 $\mathrm{min})$, cost estimation (15 $\mathrm{min}$ ) and quotation presentation (15 min), which adds up to a total of $45 \mathrm{~min}$ per customer order.

With this method, it was only necessary to include the selling price, the number of sides (cutting and welding), total stitching metres, the number of blinds and number of trips for delivery on each invoice. The number of orders, job cards and invoices are always a default value of one. The number of sides affected was assumed to be two for cutting and one for welding activities per PVC blind. The TDABC product cost could then be calculated for a PVC blind invoice.

To extrapolate the results, the revenue for all PVC blinds was expressed as a percentage of total revenue. If the turnover of PVC blinds in the month of evaluation was $4.81 \%$ of total turnover, resources consumed would be considered equal to $4.81 \%$ of total resources consumed, for example, the sales 
activity consumed R1 726.94, which is extrapolated to be R35 896.55 (R1 726.94/0.0481).

A total number of six invoices relating to PVC blind constituted the TDABC input variables. The results illustrate that $29.55 \mathrm{~h}$ were calculated with TDABC and $42.66 \mathrm{~h}$ were calculated from the job cards (Table 5). The hours calculated with TDABC relates only to the production activities (cutting, welding and stitching). If the job cards are accurate, based on hours recorded, the TDABC resource consumption is only around $69 \%\left[\frac{29.55}{42.66}\right]$ for this specific month. However, it should be noted that the TDABC calculation considers the entire value chain and it is, therefore, difficult to compare directly.

Furthermore, the hours stated on the job card would likely include time for reworks and scrapping, where applicable. The accuracy could potentially be improved with reduced estimations and more time studies. However, the narrative of this research dictates that practicality is as desirable as accuracy and that a compromise is inevitable.

In the month evaluated, resources of R90 135.98 were allocated to the sales activity using TDABC, with the result that $40 \%$ of resources were consumed (R35 896.55/R90 135.98). In Table 6 an extract is shown from the resource utilisation. In this evaluation, it is revealed that the cutting activity only utilises $31 \%$ of its resources. In contrast, the welding department uses $54 \%$ of its resources. This may be because of higher waiting times because the productivity of the other two production activities is superior.

It is highly probable that stricter time limits for product manufacturing will improve the resource utilisation. Due consideration may also be given to balancing the operations for improved productivity. In this regard, the welding activity

TABLE 5: Production hours stated on job cards compared to time-driven activitybased costing calculation.

\begin{tabular}{lccc}
\hline Description & $\begin{array}{c}\text { Revenue } \\
\text { (in Rand) }\end{array}$ & $\begin{array}{c}\text { Hours stated } \\
\text { on job cards }\end{array}$ & $\begin{array}{c}\text { Hours calculated } \\
\text { with TDABC }\end{array}$ \\
\hline Invoice 1 & 3684.21 & 6.00 & 3.95 \\
Invoice 2 & 5482.45 & 7.00 & 4.93 \\
Invoice 3 & 2105.26 & 4.00 & 2.65 \\
Invoice 4 & 7807.02 & 11.08 & 9.08 \\
Invoice 5 & 4868.42 & 9.58 & 6.03 \\
Invoice 6 & 2850.88 & 5.00 & 2.91 \\
Total revenue for PVC blinds & 26798.24 & 42.66 & 29.55 \\
only & & & - \\
Total revenue for all products & 557035.43 & - & - \\
PVC blinds as a portion of & $4.81 \%$ & - & \\
total sales & & & \\
\hline
\end{tabular}

PVC, polyvinyl chloride; TDABC, time-driven activity-based costing. is potentially a bottleneck activity in this organisation, especially if volumes should increase.

The TOC could potentially be supported with TDABC by analysing resource utilisation. Furthermore, in a manufacturing SME specialising in customer products, it is plausible that flexing of production headcount in response to the order book could lower wage costs, thereby improving consumption of resources. A further revelation is that the fitting activity has consumed $104 \%$ of resources which, in theory, should be unlikely (Table 6). This can probably be attributed to either an overestimation of work step times or, in fact, an above-average delivery month with shorter trips to customers. It was noted by the owner during the later evaluation phase that one person (out of two) has left the fitting department and was not replaced. One person from the cutting department was asked to assist with fitting functions on an ad hoc basis.

In addition to evaluating the resources consumed for activities, it was also possible to analyse the income statement and express resources consumed as a percentage by resource group. It was calculated that only $40 \%$ of rental and insurance costs were converted into sales (Table 7). The reason for the low consumption rate was the high rental costs associated with the stitching department because of unused space. The owner indicated that some of the space would be subject to subletting at a later stage to recover some of the cost. The income statement for a later month confirmed an increase in rental income that would help to negate unused capacity. An increase in total sales and/or lowering of fixed costs could allow the organisation to improve the percentage consumed for all resource categories.

\section{Ethical consideration}

The research was conducted after ethical clearance was obtained from the Nelson Mandela University research committee (Ref: H-15-BES-ACC-020). Permission to conduct the research at the manufacturing SME used for the case study was granted on condition of anonymity and that no sensitive financial information is published. The manuscript

TABLE 7: Resource utilisation by resource group.

\begin{tabular}{lccc}
\hline Resource & $\begin{array}{c}\text { Expenditure } \\
\text { (in Rand) }\end{array}$ & $\begin{array}{c}\text { Consumed } \\
\text { (in Rand) }\end{array}$ & Consumed (\%) \\
\hline Salaries and wages & 206488.26 & 90622.27 & 44 \\
Energy & 6383.93 & 2801.74 & 44 \\
Telephone expense & 4099.68 & 1767.86 & 43 \\
Insurance & 9210.85 & 3700.67 & 40 \\
Rental & 36062.00 & 14488.72 & 40 \\
Vehicle expense & 21131.64 & 11852.43 & 56 \\
Overheads & 65962.62 & 29151.14 & 44 \\
Available resources & 349338.98 & 154384.83 & 44 \\
\hline
\end{tabular}

TABLE 6: Resource utilisation for the six different activities.

\begin{tabular}{|c|c|c|c|c|c|c|}
\hline Resource & Cutting & Welding & Stitching & Sales & Admin & Fitting \\
\hline Expenditure (In Rand) & 60999.81 & 29920.76 & 67931.87 & 90135.98 & 74320.46 & 26030.10 \\
\hline Consumed resources (In Rand) & 18868.43 & 16196.38 & 26363.87 & 35896.55 & 29885.40 & 27174.20 \\
\hline Resource utilisation & $31 \%$ & $54 \%$ & $39 \%$ & $40 \%$ & $40 \%$ & $104 \%$ \\
\hline
\end{tabular}


was submitted for publishing only after written approval (available on request) was obtained from the SME owners to confirm their satisfaction with the content.

\section{Results}

The primary objective of the research was to adapt the generic TDABC framework as proposed by Kaplan and Anderson (2003) to suit SMEs with resource constraints. The implementation steps for the study differ from the generic steps described by Kaplan and Anderson (2003) in two areas, namely, product focus and combination of work steps (or sub-activities). The generic implementation steps were found to be inappropriate, at least for an SME with multiple products and limited resources. The research confirms the findings from published literature that TDABC implementation should be adapted where constraints exists, especially for SMEs and other resource-constrained settings (Balakrishnan et al. 2015; Fladkjaer \& Jenson 2011; Ganorkar et al. 2018; Somapa et al. 2012; Souza et al. 2010; Stouthuysen et al. 2014).

It was found that TDABC implementation for the case SME could only be achieved if the focus was on a single product that is frequently sold. Although the use of a single product is very practical, it is probable that evaluating two or more products may improve accuracy. This may apply to many SMEs with limited resources and varied product ranges. In contrast with the view of Stouthuysen et al. (2014:295), the TDABC analysis in this case considered all costs, including fixed and shared overheads. To achieve maximum accuracy, it was found to be beneficial to evaluate the entire value chain from receipt of the order to delivery and follow-up with the customer to establish all the work steps. The consumption of resources (in hours) was compared to the hours stated on the job cards (refer to Appendix 3). The calculation revealed under-absorption of $13 \mathrm{~h}$ (or 31\%) compared to the job card hours. It may be worthwhile to investigate measures to further improve accuracy. Physical measurement of activities was not always possible, but in many cases an approximation was appropriate by consulting the relevant staff members or by direct observation. In the case of production activities, a time study was conducted to measure the duration of the work steps. It was not practical to include all the work steps in the TDABC equations, as this would have unnecessarily complicated the costing system. The work steps were, therefore, grouped within the activities, which resulted in simpler time equations.

The use of a single product and combining work steps meant TDABC calculation for a single month could be derived from the input of six invoices only. It was suggested by Souza et al. (2010:68) that made-to-order (custom) organisations cannot generalise the findings from TDABC across entire product ranges. This research suggests that although it cannot be known if TDABC results can be generalised, there is still a benefit from partially implementing TDABC. The use of Microsoft Excel for Mac for the TDABC calculation was considered more than sufficient for the required implementation. In contrast to the view of Stout and Propri (2011:2), it is unlikely that investment in a dedicated enterprise resource planning (ERP) system for TDABC would have derived more benefits. Although it is relatively easy and quick to input into the TDABC spread sheet, there is also the risk of changes in product mix, which may result in too little, or even no, data to capture. It may, therefore, be worthwhile to consider evaluating two or more key products, rather than just a single product.

It was evident from the evaluation of the TDABC data that it was possible to identify two key problems in the factory, namely, high expenditure and unused capacity. Furthermore, the TOC can potentially be managed with TDABC, which may improve order turnaround time. The high salary and wage costs in the admin activity and the high rental costs in the stitching activity were two examples of how the TDABC evaluation has highlighted high expenditure before resource consumption were considered. The low resource utilisation in the cutting activity has revealed a higher than optimum headcount in this area. In this regard, the findings have illustrated potential use for SMEs with resource constraints by adapting the generic TDABC proposed in literature to ensure implementation is practical (Figure 3).

\section{Conclusion}

Although the implementation of TDABC is described in published literature with the use of the six-step process outlined by Kaplan and Anderson (2003), it was found that applying the TDABC implementation steps under status quo to an SME with limited resources is not practical. The current study has shown that it is possible to implement TDABC and to accrue benefits by highlighting specific areas of concern, permitting that due consideration is awarded to the limitations of the organisation. Furthermore, the analysis of relevant activities may enable SMEs to consider the TOC for more effective operations. The current study's results contribute to existing literature by providing a revised framework for

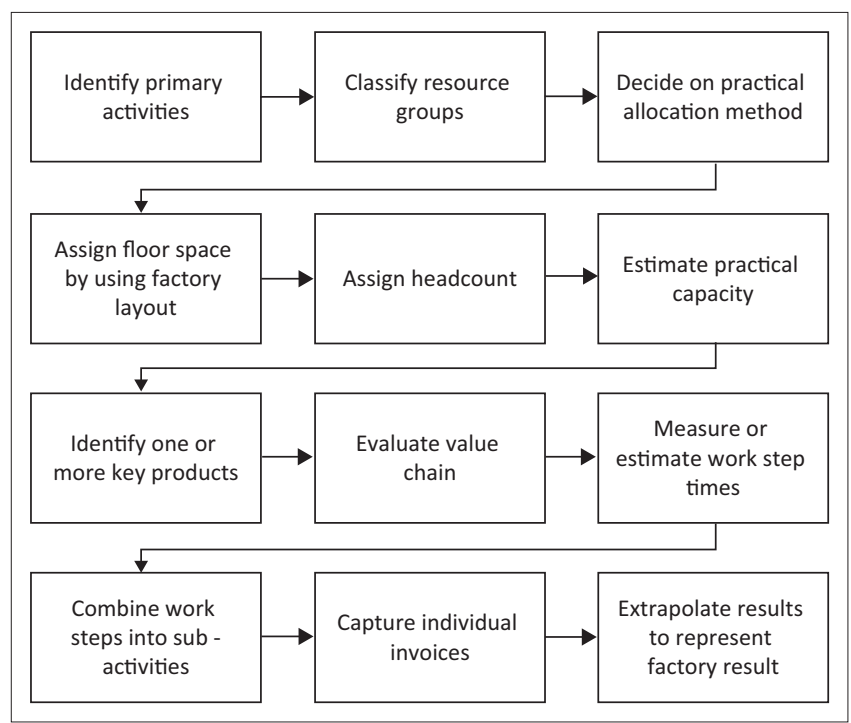

FIGURE 3: Proposed modified framework for time-driven activity-based costing implementation at small and medium enterprises. 
TDABC implementation in a SME with resource constraints. The strategy towards TDABC implementation at SMEs with resource constraints could be informed by the developed framework. It is worth noting that benefit could be derived from TDABC directly after implementation. Most SMEs will likely not have a dedicated person to perform the cost accounting function on a continuous basis. Therefore, consideration may have to be given to using TDABC only on an ad hoc basis (e.g. annually) to evaluate costs because of the quick and simple implementation time.

As a result of evaluation of a small percentage of products, the system cannot claim to be $100 \%$ accurate, but may merely serve as a reasonable approximation of TDABC product cost and unused capacity. The impact that other products may have on the evaluation is unknown and this is a weakness of the current study. As such, the results of this study should not be generalised to all SMEs. Furthermore, based on the reported resource utilisations, estimations for work step times are potentially underestimated and, therefore, resource utilisation could have been under-reported. In contrast, it is possible that the arbitrary $80 \%$ assumed for practical capacity may have been overstated, which may need to be adjusted by using appropriate measures. In addition, focusing on only select products does not allow for evaluation of product mix decision. This may be negated by performing multiple ad hoc TDABC calculations for a different product.

\section{Recommendations and suggestions for future research}

It is recommended that owners and managers of SMEs adopt the framework for TDABC implementation, if limited resources are available, for a focused implementation and continuous evaluation of costing data. Furthermore, it is proposed that SMEs with resource constraints consider TDABC analysis on an ad hoc basis only, because benefits could still be accrued in this manner. It is recommended that future research focus on the continuous evaluation of the implementation framework, described in this article, to establish its effectiveness over several months or longer. Furthermore, it is prescribed that this partial product evaluation is compared with a comprehensive evaluation for the complete product range to establish to what extent TDABC accuracy is affected. In addition, it is proposed that further research establishes the suitability of ad hoc TDABC evaluations for SMEs.

\section{Acknowledgements}

The authors are grateful for the input from owners of the SME for the opportunity to conduct the case study at their organisation and for the time that was set aside for this research. Furthermore, the contribution of Aina Kapulwa, the research assistant, in collecting documentary evidence, conducting the time studies and engaging with the SME owners is acknowledged. This study was funded through a postgraduate bursary for PhD studies by Nelson Mandela University.

\section{Competing interests}

The views expressed in this article are our own and do not necessarily reflect the official position of the listed institutions. The authors declare that they have no financial or personal relationships that may have inappropriately influenced them in writing this article.

\section{Authors' contribution}

A.R. constructed the article from the research conducted during the PhD study. H.F. and L.J.E. were responsible for academic insight and review.

\section{References}

Akyol, D.E., Tuncel, G. \& Bayhan, G.M., 2007, 'A comparative analysis of activity-based costing and traditional costing', International Journal of Industrial and Manufacturing Engineering 1(3), 136-139.

Askarany, D. \& Franklin-Smith, A.W., 2014, 'Cost benefit analyses of organic waste composting systems through the lens of time driven activity-based costing', Journal of Applied Management Accounting Research 12(2), 59-73.

Balakrishnan, K., Goico, B. \& Arjmand, E.M., 2015, 'Applying cost accounting to operating room staffing in Otolaryngology: Time-driven activity-based costing and outpatient adenotonsillectomy', Otolaryngology-Head and Neck Surgery 152(4), 684-690. https://doi.org/10.1177/0194599814568273

Drury, C., 2015, Management and cost accounting, 9th edn., Pat Bond, London.

Fladkjaer, H. \& Jensen, E., 2011, The ABC-paradox: Is time driven $A B C$ relevant for small and medium sized enterprises (SME)? Aalborg University, Department of Business and Management, Working Paper, 2, pp. 1-23.

Ganorkar, A.B, Lakhe, R.R. \& Agrawal, K.N., 2018, 'Implementation of TDABC in SME: A case study', Journal of Corporate Accounting and Finance 29(2), 87-113. https:// doi.org/10.1002/jcaf.22327

Goldratt, E.M., 1990, What is this thing called the Theory of Constraints? North River Press, Croton-on-Hudson, NY.

Ingle, M., 2014, 'An analysis of factors impeding SMME performance in South Africa', Africa Insight 44(2), 37-50.

Kaplan, R.S. \& Anderson, S.R., 2003, Time-driven activity-based costing, viewed 22 May 2018, from https://papers.ssrn.com/sol3/papers.cfm?abstract_id $=485443$

Mabin, V., 2015, 'Goldratt's "Theory of Constraints" thinking processes: A systems methodology linking soft with hard', Review of Business Management 15(46), 129-148.

Musov, M., 2017, Time-driven activity-based costing: Potential for application at the SMEs in Poland and Bulgaria, viewed 20 September 2018, from https://ssrn.com/ abstract $=2924495$

National Credit Regulator (NCR), 2011, Literature review on small and medium enterprises' access to credit and support in South Africa, viewed 22 May 2018, from
http://www.ncr.org.za/pdfs/Literature $\% 20$ Review $\% 20$ on $\% 20$ MEE $\% 20$ Access $\% 20$ to $\% 20$ Credit $\% 20$ in $\% 20$ South\%20Africa_Final\%20Report_NCR_Dec\%202011.pdf

Öker, F. \& Adıgüzel, H., 2010, 'Time-driven activity-based costing: An implementation in a manufacturing company', The Journal of Corporate Accounting \& Finance 22(1), 75-92. https://doi.org/10.1002/jcaf.20646

Olawale, F. \& Garwe, D., 2010, 'Obstacles to the growth of new SME's in South Africa: A principal component analysis approach', African Journal of Business Management 4(5), 729-738.

Pegels, C.C. \& Watrous, C., 2005. 'Application of the Theory of Constraints to a bottleneck operation in a manufacturing plant', Journal of Manufacturing Technology Management 16(3), 302-311. https://doi.org/10.1108/17410380510583617

Reynolds, A. \& Van der Poll, H.M., 2015, 'ABC implementation in the Nelson Mandela Bay Metropole: How far should manufacturing organisations go?', Southern African Business Review, Special Edition Accounting Research 19(2), 118-136.

Shea, V.J., Waldrup, B.E., Xu, H. \& Williamson, S., 2018, 'Error rate impacts on decision efficacy', Quarterly Review of Business Disciplines 5(1), 59-71.

Siquenza-Guzman, L., Van der Abbeele, A. \& Cattrysse, D., 2014, 'Time-driven activitybased costing systems for cataloguing processes: A case study', Liber Quarterly 23(3), 160-186.

Somapa, S., Cools, M. \& Dullaert, W., 2012, 'Unlocking the potential of time-driven activity-based costing for small logistics companies', International Journal of Logistics: Research and Applications 15(5), 303-322. https://doi.org/10.1080/136 75567.2012.742043

Souza, A.A.D., Avelar, E.A., Machado Boina, T. \& Raimundini, S.L., 2010, 'Análise Da Aplicabilidade do time-driven activity-based costing Em Empresas de Produção por Encomenda [Analysis of the applicability of the time driven activity-based costing in make-to-order companies]', Revista Universo Contábil 6(1), 67-84.

Stout, D.E. \& Propri, M.L., 2011, 'Implementing time-driven activity-based costing at a medium-sized electronics company', Management Accounting Quarterly 12(3), $1-11$.

Stouthuysen, K., Schierhout, K., Roodhooft, F. \& Reusen, E., 2014, 'Time-driven activity-based costing for public services', Public Money \& Management 34(4), 289-296. https://doi.org/10.1080/09540962.2014.920202 


\section{Appendix 1}

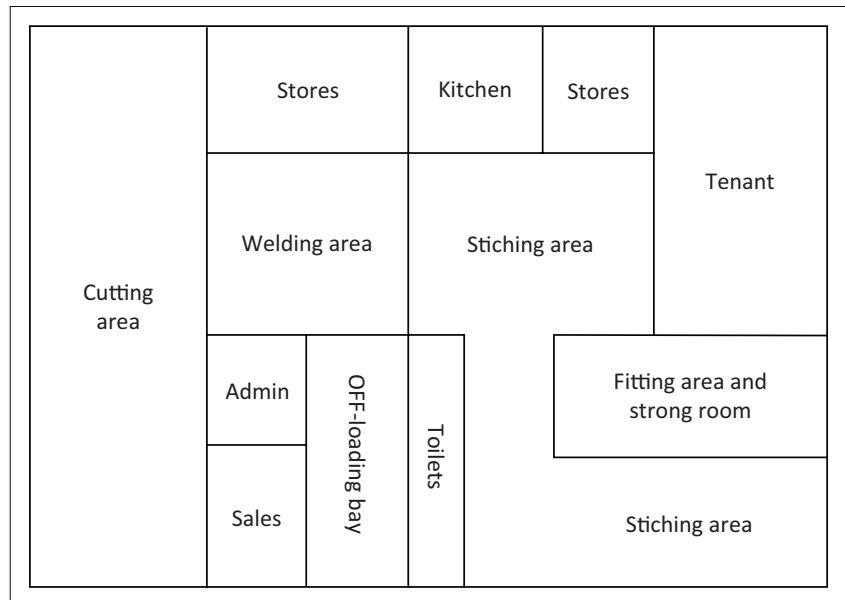

FIGURE 1-A1: Factory layout and primary activity areas. 


\section{Appendix 2}

TABLE 1-A2: Work steps for polyvinyl chloride blinds.

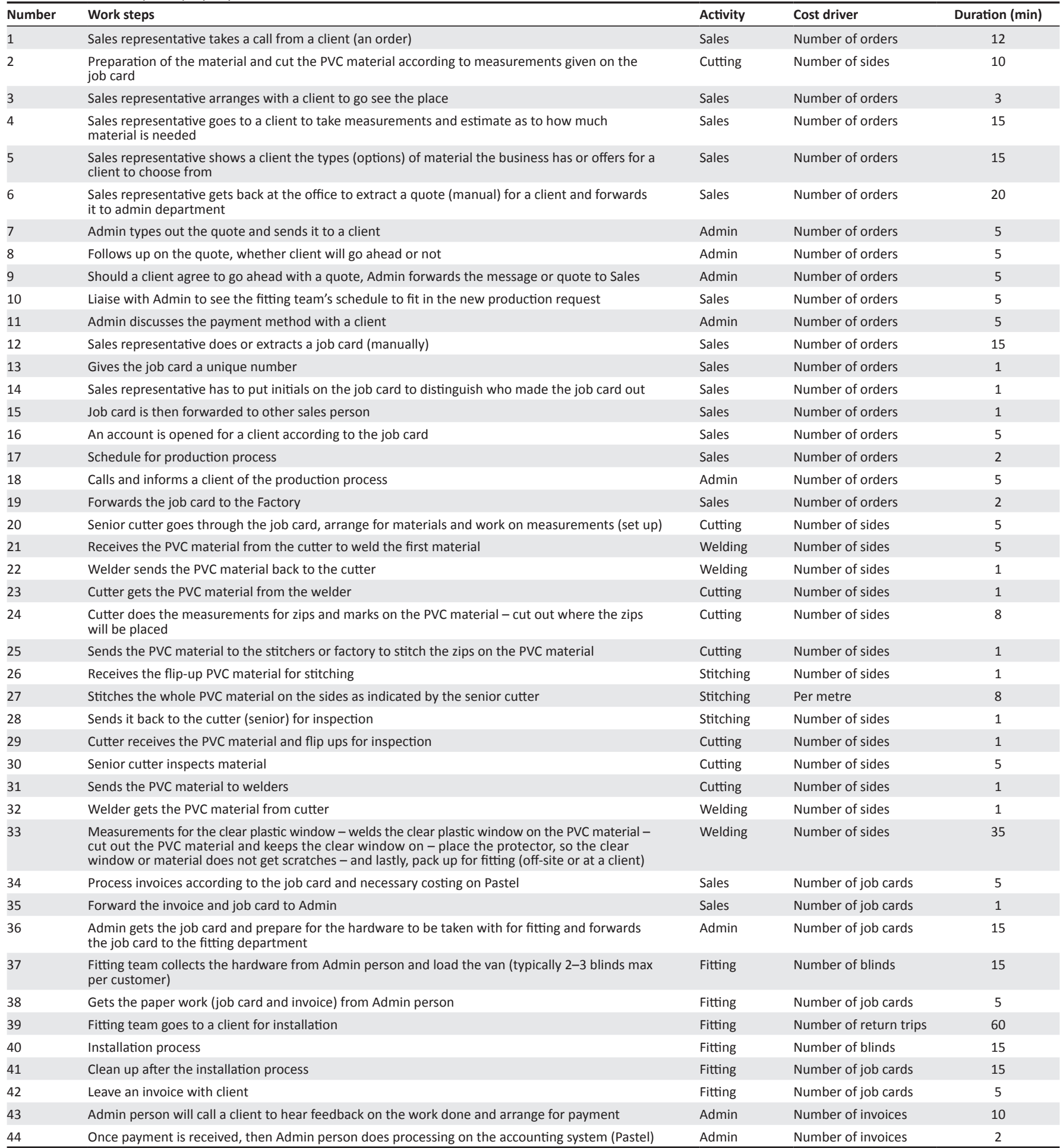

PVC, polyvinyl chloride, min, minutes. 


\section{Appendix 3}

TABLE 1-A3: Calculations for resource consumption

\begin{tabular}{|c|c|c|c|c|c|c|c|c|c|c|c|c|}
\hline Process & Department & $\begin{array}{l}\text { Rate per } \\
\text { min }\end{array}$ & Cost driver & Min & Cost & Invoice 1 & Invoice 2 & Invoice 3 & Invoice 4 & Invoice 5 & Invoice 6 & Total \\
\hline Revenue & - & - & - & - & - & R 3684.21 & R 5482.45 & R 2105.26 & R 7807.02 & R 4868.42 & R 2850.88 & R 26798.24 \\
\hline Order receipt & Sales & R 2.79 & Number of orders & 45 & R 125.75 & 1.0 & 1.0 & 1.0 & 1.0 & 1.0 & 1.0 & 6.0 \\
\hline Quotation to customer & Sales & R 2.79 & Number of orders & 20 & R 55.89 & 1.0 & 1.0 & 1.0 & 1.0 & 1.0 & 1.0 & 6.0 \\
\hline Quotation to customer & Admin & R 4.61 & Number of orders & 20 & R 92.16 & 1.0 & 1.0 & 1.0 & 1.0 & 1.0 & 1.0 & 6.0 \\
\hline Job card preparation & Sales & R 2.79 & Number of orders & 32 & R 89.42 & 1.0 & 1.0 & 1.0 & 1.0 & 1.0 & 1.0 & 6.0 \\
\hline Job card preparation & Admin & R 4.61 & Number of orders & 5 & R 23.04 & 1.0 & 1.0 & 1.0 & 1.0 & 1.0 & 1.0 & 6.0 \\
\hline Preparation & Cutting & R 1.51 & Number of sides & 15 & R 22.69 & 2.0 & 2.0 & 2.0 & 8.0 & 4.0 & 2.0 & 20.0 \\
\hline Welding & Welding & R 1.86 & Number of sides & 40 & R 74.21 & 1.0 & 1.0 & 1.0 & 4.0 & 2.0 & 1.0 & 10.0 \\
\hline Material movement & Welding & R 1.86 & Number of sides & 2 & R 3.71 & 1.0 & 1.0 & 1.0 & 4.0 & 2.0 & 1.0 & 10.0 \\
\hline Material movement & Cutting & R 1.51 & Number of sides & 4 & R 6.05 & 1.0 & 1.0 & 1.0 & 4.0 & 2.0 & 1.0 & 10.0 \\
\hline Cutting & Cutting & R 1.51 & Number of sides & 8 & R 12.10 & 2.0 & 2.0 & 2.0 & 8.0 & 4.0 & 2.0 & 20.0 \\
\hline Material movement & Stitching & R 1.68 & Number of sides & 2 & R 3.37 & 1.0 & 1.0 & 1.0 & 4.0 & 2.0 & 1.0 & 10.0 \\
\hline Stitching & Stitching & R 1.68 & Per metre & 8 & R 13.48 & 16.6 & 24.0 & 6.9 & 16.1 & 19.2 & 8.8 & 91.6 \\
\hline Quality check & Cutting & R 1.51 & Number of sides & 5 & R 7.56 & 2.0 & 2.0 & 2.0 & 8.0 & 4.0 & 2.0 & 20.0 \\
\hline Invoicing & Sales & R 2.79 & Number of job cards & 6 & R 16.77 & 1.0 & 1.0 & 1.0 & 1.0 & 1.0 & 1.0 & 6.0 \\
\hline Fitting & Admin & R 4.61 & Number of job cards & 15 & R 69.12 & 1.0 & 1.0 & 1.0 & 1.0 & 1.0 & 1.0 & 6.0 \\
\hline Fitting & Fitting & R 1.61 & Number of blinds & 30 & R 48.42 & 1.0 & 1.0 & 1.0 & 4.0 & 2.0 & 1.0 & 10.0 \\
\hline Fitting & Fitting & R 1.61 & Number of job cards & 25 & R 40.35 & 1.0 & 1.0 & 1.0 & 1.0 & 1.0 & 1.0 & 6.0 \\
\hline Fitting & Fitting & R 1.61 & Number of trips & 60 & R 96.84 & 1.0 & 1.0 & 1.0 & 1.0 & 1.0 & 1.0 & 6.0 \\
\hline Follow-up & Admin & R 4.61 & Number of invoices & 12 & R 55.30 & 1.0 & 1.0 & 1.0 & 1.0 & 1.0 & 1.0 & 6.0 \\
\hline Total minutes & All & - & - & - & - & 506.80 & 566.00 & 429.20 & 904.80 & 661.60 & 444.40 & 3512.80 \\
\hline Total minutes & Production & - & - & - & - & 236.80 & 296.00 & 159.20 & 544.80 & 361.60 & 174.40 & 1772.80 \\
\hline Resources consumed & Sales & R 2.79 & - & - & - & R 287.82 & R 287.82 & R 287.82 & R 287.82 & R 287.82 & R 287.82 & R 1726.94 \\
\hline Resources consumed & Admin & R 4.61 & - & - & - & R 239.62 & R 239.62 & R 239.62 & R 239.62 & R 239.62 & R 239.62 & R 1437.75 \\
\hline Resources consumed & Cutting & R 1.51 & - & - & - & R 90.77 & R 90.77 & R 90.77 & R 363.09 & R 181.55 & R 90.77 & R 907.74 \\
\hline Resources consumed & Welding & R 1.86 & - & - & - & R 77.92 & R 77.92 & R 77.92 & R 311.67 & R 155.84 & R 77.92 & R 779.19 \\
\hline Resources consumed & Stitching & R 1.68 & - & - & - & R 227.11 & R 326.85 & R 96.37 & R 230.48 & R 265.53 & R 121.98 & R 1268.33 \\
\hline Resources consumed & Fitting & R 1.61 & - & - & - & R 185.61 & R 185.61 & R 185.61 & R 330.86 & R 234.03 & R 185.61 & R 1307.32 \\
\hline
\end{tabular}

